\title{
Low scaling excited state calculation using block interaction product state
}

\author{
Ke Wang and Haibo Ma* \\ School of Chemistry and Chemical Engineering, Jiangsu Key Laboratory of Vehicle \\ Emissions Control, Nanjing University, Nanjing 210023, China \\ E-mail: haibo@nju.edu.cn
}

\begin{abstract}
We develop an automatic and efficient scheme for the accurate construction of the bases for excitonic models, which can enable "black-box" excited state structure calculations for large molecular systems. These new and optimized bases, which are named as block interaction product state (BIPS), can be expressed as the direct products of the local states for each chromophore. Each chromophore's local states are selected by diagonalization of its reduced density matrix (RDM), which is obtained by quantum chemical calculation of the small subsystem composed of the chromophore and its nearest neighbors. We implemented BIPS framework with fragment-based calculations considering 2-body and 3-body interactions. Test calculations for 8 different molecular aggregates demonstrate that this framework provides accurate description of not only the excitation energies, but also the first-order wavefunction properties (dipole moment and transition dipole moment) of the low-lying excited states at a low-scaling computational cost.
\end{abstract}

The accurate description of the electronic structure for the electronic excited states is the key foundation for a reliable theoretical interpretation of experimental spectroscopy and dynam- 
ics as well as other properties in photochemistry and photophysics. ${ }^{1-3}$ However, when dealing with large photoactive systems, such as molecular aggregates in organic semiconductor materials or biological light harvesting systems, conventional excited-state quantum chemical methods like full configuration interaction (FCI), complete active space self-consistent field (CASSCF), configuration interaction singles (CIS), or time-dependent density functional theory (TDDFT), can hardly fulfil the appeal of excited state calculation due to the high-scaling growth of the computational cost with the increasing systems size. ${ }^{4}$ Nowadays, it is usually considered that the upper limit for the applicability of conventional excited state quantum chemical methods is merely about 150-200 atoms, if no further approximations are introduced. ${ }^{5}$ Encouragingly, inspired by the great success of developed low-scaling quantum chemistry methods for ground state in the last few decades, ${ }^{6-10}$ many low-scaling excited state electronic structure methods have been proposed recently. Developed lowscaling excited state methods can be generally classified into three categories. The first category combines the local excitation approximation (LEA), in which the electronic excitation is restricted to only one specific region, with the different types of low-scaling fragmentbased approach, such as fragment molecular orbital (FMO), ${ }^{11,12}$ generalized energy-based fragment (GEBF) ${ }^{13}$ electrostatically embedded generalized molecular fractionation method $(\text { EE-GMF })^{14}$ and other general embedding strategies. ${ }^{15,16}$ It has been shown that these methods can successfully give reasonable descriptions for large systems having only single local excitations, such as interpreting enhanced molecular fluorescence in crystal environments. ${ }^{17}$ As for describing more general delocalized or multiple excitation behaviors in large molecules, one can resort to the second category of low-scaling excited state methods based on local correlation approximation (LCA). In this kind of approach, based on the local feature of the density matrix or molecular orbitals, it is assumed that the excitation could be approximately described by considering only neighboring electronic couplings. The LCA scheme has been successfully implemented in various $a b$ initio levels of TDDFT ${ }^{18-24}$ and wavefunction theory (WFT) ${ }^{22,25-35}$ and helped describe non-local excitations such as simu- 
lating the photo-absorption spectrum in metal clusters. ${ }^{36}$ Another efficient LCA strategy to inexpensively treat excited states is approximating those as poles of dynamical polarizability evaluated by divide-and-conquer (DC) approaches, proposed and implemented recently by Nakai and coworkers. ${ }^{37-39}$ The third category of low-scaling excited state methods adopts excitonic model Hamiltonians, which are widely used in material science and computational biology. ${ }^{40-45}$ By applying the exciton concept to approximate local excited states of chromophores, one can easily characterize the collective excitations of molecule aggregates estimating the inter-chromophore coupling terms in various approximation ways. ${ }^{46-49}$ Despite of the great success of its wide applications in excited state problems, empirical excitonic model still suffers from a few drawbacks. First, the definition of model basis and the construction of the model is not automatic. The choice of its parameter values sometimes relies heavily on experimental fitting or experience setting, which greatly restricts its further extension to new systems. Secondly, the accurate derivation of parameter values from quantum chemical calculations is challenging, owing to the co-existence of short-range exchange interactions and long-range Coulomb interactions. ${ }^{50-52}$

To construct the basis for the excitonic model, a widely used and straightforward strategy hinted by the numerical renormalization group $(\mathrm{NRG}),{ }^{53}$ is to take the direct product of energetically low-lying local states for each subsystem as the effective bases. This approach is adopted in contractor renormalization group (CORE), ${ }^{54}$ active space decomposition method $(\mathrm{ASD})^{55,56}$ and renormalization group method (REM), ${ }^{57-61}$ where the low-lying states of a system can be expressed as the linear superposition of the effective base configurations, which are the direct products of several energetically low-lying local subsystem wavefunctions. By only taking a small number of effective bases, low-scaling computational efficiency can be easily achieved, which makes these methods promising for calculating excited states of large molecular systems. However, the selection of these effective bases still depends on personal experience, and one may hardly generate the proper bases automatically when more than one excited state or charge-transfer (CT) state may be involved. Additionally, in 
these methods, local wavefunctions at each chromophore are usually computed in isolation and no interactions with surrounding subsystems (i.e., the environment) is considered, thus limiting the accuracy of the total excitation energy and wavefunction. Recently, Nishio and Kurashige ${ }^{62}$ proposed an improved strategy to account for the inter-chromophore effect in their rank-one excitonic basis. In given approach, local states are iteratively optimized under the potential produced by other subsystems. Nevertheless, the automatic selection of the numbers and types of rank-one basis for complicated excitation systems is still unavailable.

According to quantum information theory, the most efficient and optimal way to construct a truncated Hilbert space for a subsystem is to select the states which maximize the subsystem's entanglement with its environment. ${ }^{63}$ This can be achieved by preserving the eigenstates of the subsystem's reduced density matrix (RDM) with leading large eigenvalues, which contributes to the great success of density matrix renormalization group (DMRG) ${ }^{64}$ for strongly correlated systems. However, how to utilize the system-environment entanglement to construct the basis for excitonic model is unexplored yet, due to the large size of the targeted system.

In this work, we introduced a robust automatic scheme for the accurate construction of the bases for excitonic models, thus enabling "black-box" low-scaling excited state structure calculations in large systems. The new bases are named as block interaction product state (BIPS), where chromophore's local states are obtained from the diagonalization of its RDM, which is calculated from a solution of the small-sized chromophore cluster containing only the monomer of interest and its neighbors. Results from the proof-of-principle test calculations for 8 different kinds of one dimensional (1D) molecular aggregates suggest that our new scheme can accurately describe not only the excitation energies with the chemical accuracy $\left(\sim 1 \mathrm{kcal} \mathrm{mol}^{-1}\right)$, but also wavefunction properties (dipole moment and transition dipole moment) of the low-lying delocalized electronic states in large systems. This provides an automatic and accurate low-scaling electronic structure approach for excited state studies in various large chemical systems, which will benefit future theoretical studies of photophysics 
and photochemistry in chemistry, biology and material science.
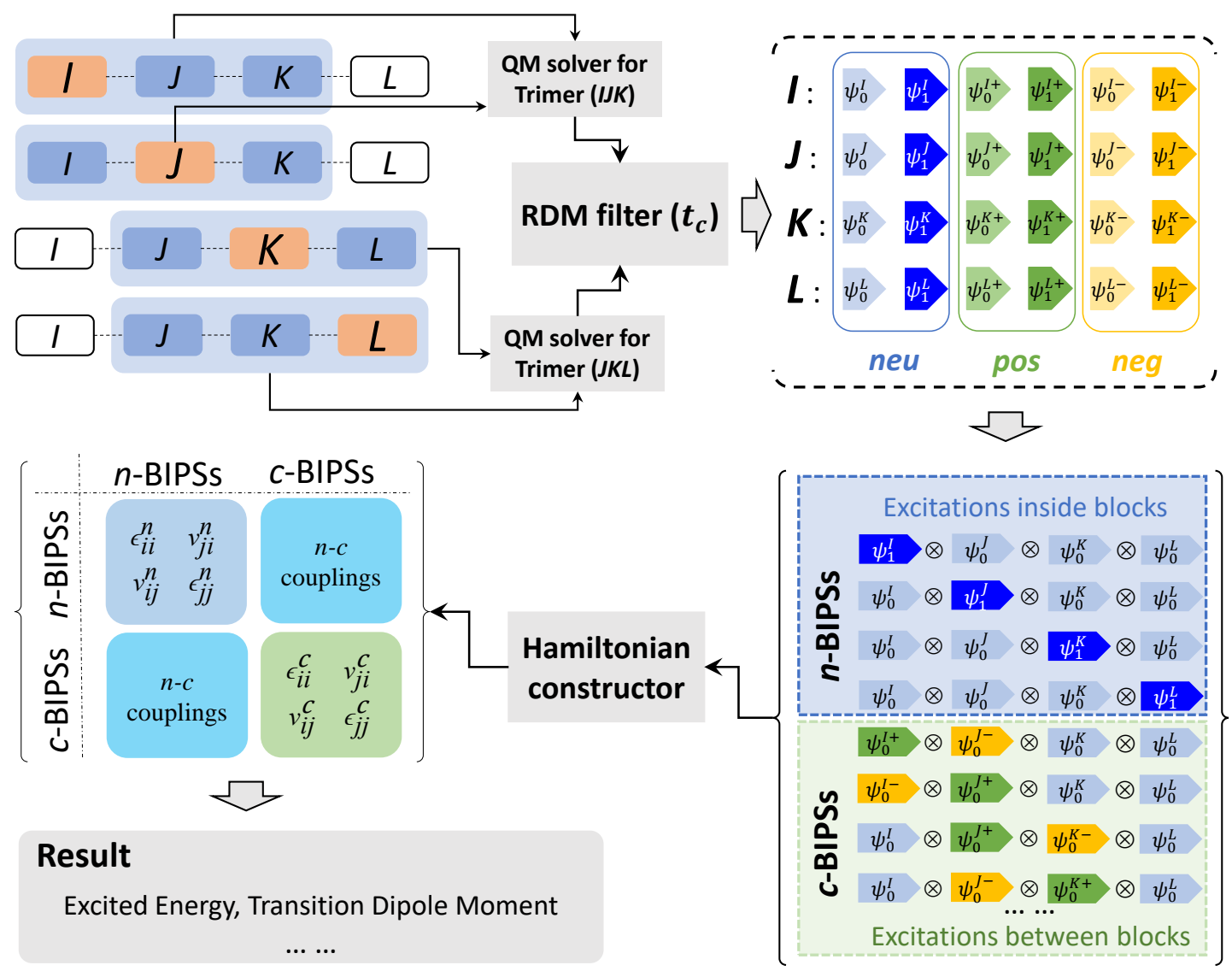

Figure 1: Schematic diagram for the low-scaling excited state calculation using BIPS (taking a tetramer of IJKL as an example).

In order to calculate low-scaling excited states with BIPS, the whole excited state system is divided into $N$ block monomers (i.e., chromophores), as illustrated in Figure 1. For computational simplicity, an orbital localization procedure is implemented after a global Hartree-Fock calculation to obtain a set of orthogonal localized molecular orbitals (OLMOs), which are then assigned to each block for constructing the local states. Taking block $J$ in the 1D tetramer aggregate in Figure 1 as an example, it gets together with its neighboring environment, blocks $I$ and $K$, forming a trimer block cluster. Hereby, the interested state $\left|\psi^{*}\right\rangle$ $\left(\mathrm{T}_{1}\right.$ or $\left.\mathrm{S}_{1}\right)$ of this block cluster (trimer $\left.I J K\right)$ can be obtained through a standard quantum chemical calculation, i.e.

$$
\widehat{H}_{I J K}\left|\psi^{*}\right\rangle=\epsilon\left|\psi^{*}\right\rangle
$$


Accordingly, the density operator for state $\left|\psi^{*}\right\rangle$ can be expressed as

$$
\widehat{\rho}=\left|\psi^{*}\right\rangle\left\langle\psi^{*}\right|
$$

Since OLMOs are used, which can be assigned to each monomer respectively, the $\left|\psi^{*}\right\rangle$ can be re-expressed as the superposition of the product states of wavefunctions for the subsystem and its environment (block $I$ and $K$ ),

$$
\left|\psi^{*}\right\rangle=\sum_{i, j} c_{i j}\left|\psi_{i}^{\mathrm{S}} \psi_{j}^{\mathrm{E}}\right\rangle
$$

Here $\psi_{i}^{\mathrm{S}}$ and $\psi_{j}^{\mathrm{E}}$ represent the possible local configurations for the system and its environment respectively. $c_{i j}$ is the configuration coefficient in the standard quantum chemical calculation of the cluster. Thereby, Equation (2) becomes

$$
\widehat{\rho}=\sum_{i, j} \sum_{i^{\prime}, j^{\prime}} c_{i j} c_{i^{\prime} j^{\prime}}\left|\psi_{i}^{\mathrm{S}} \psi_{j}^{\mathrm{E}}\right\rangle\left\langle\psi_{i^{\prime}}^{\mathrm{S}} \psi_{j^{\prime}}^{\mathrm{E}}\right|
$$

After summing all items belonging to the environment (block $I$ and $K$ ), the reduced density operator $\widehat{\rho_{\mathrm{S}}}$ is obtained for the system (block $J$ ) by

$$
\widehat{\rho_{\mathrm{S}}}=\operatorname{Tr}_{E}(\widehat{\rho})=\sum_{j}\left\langle\psi_{j}^{\mathrm{E}} \mid \psi^{*}\right\rangle\left\langle\psi^{*} \mid \psi_{j}^{\mathrm{E}}\right\rangle=\sum_{i, i^{\prime}} c_{i i^{\prime}}^{\prime}\left|\psi_{i}^{\mathrm{S}}\right\rangle\left\langle\psi_{i^{\prime}}^{\mathrm{S}}\right|
$$

where

$$
c_{i i^{\prime}}^{\prime}=\sum_{j} c_{i j} c_{i^{\prime} j}
$$

The coefficients $\left\{c_{i i^{\prime}}^{\prime}\right\}$ constitute the RDM $\mathbf{D}_{J}$ for block $J$. By diagonalizing $\mathbf{D}_{J}$, one can gain a series of RDM eigenvectors with eigenvalues $\lambda_{i}$, which satisfy the following normalization condition,

$$
\sum_{i} \lambda_{i}=1
$$


To build an efficient Hilbert space with limited size, not all RDM eigenvectors are necessarily preserved, thus only these with eigenvalues larger than certain truncation threshold $t_{c}$ will be included. Contrary to traditional empirical treatments, such reserved states not only are automatically determined but also consider environmental effects. According to the net charge in the block states, these reserved states in block $J$ can be classified into three groups: the neutral states (neu), the positively charged states (pos) and the negatively charged states $(n e g)$ :

$$
\begin{gathered}
\text { neu : }\left|\psi_{0}^{J}\right\rangle,\left|\psi_{1}^{J}\right\rangle,\left|\psi_{2}^{J}\right\rangle, \cdots \\
\text { pos : }\left|\psi_{0}^{J^{+}}\right\rangle,\left|\psi_{1}^{J^{+}}\right\rangle,\left|\psi_{2}^{J^{+}}\right\rangle, \cdots \\
\text { neg }:\left|\psi_{0}^{J^{-}}\right\rangle,\left|\psi_{1}^{J^{-}}\right\rangle,\left|\psi_{2}^{J^{-}}\right\rangle, \cdots
\end{gathered}
$$

After performing the above procedures for other blocks, the reserved states can be obtained for each block. Consequently, one can easily construct the BIPSs for the whole system by taking the direct products of these optimally-selected local states for each block. All the generated BIPSs can be classified into two groups: $c$-BIPS which stands for the excitation between blocks, and $n$-BIPS (only neu) which indicates the excitation inside the block. In the scheme here proposed, the multi-excitations in many blocks are omitted.

The final step comprises the construction of the CI Hamiltonian matrix in the space spanned by the selected BIPSs, thereon a diagonalization is performed. Based on the principle of diabatization approach, it is simple to obtain the Hamiltonian by considering $n$-body interactions $(n=2,3 \ldots)$. A detailed algorithm for this purpose is provided in the Supporting Information. In this work, BIPS(2) stands for BIPS framework considering up to 2-body interactions, whereas BIPS(3) represents BIPS framework with considerations of up to 3body interactions. Results from proof-of-principle test calculations using 8 different kinds of 1D molecular aggregates at the simplest excited state calculation level of CIS/STO-6G are given below. Standard CIS/STO-6G results are utilized as reference for comparison. 
For each aggregate, the geometry of molecular monomer (except for hydrogen molecule with the bond length set to be $0.7414 \AA$ ) is optimized by density functional theory (DFT) at M06-2X/6-31G(d) ${ }^{65}$ level using the Gaussian 16 package. ${ }^{66}$ All related CIS calculations, together with BIPS(2) and BIPS(3) calculations at CIS level are implemented using our in-house code. Related orbital integrals and OLMO generation are obtained using OPENMoLCAS package. ${ }^{67,68}$
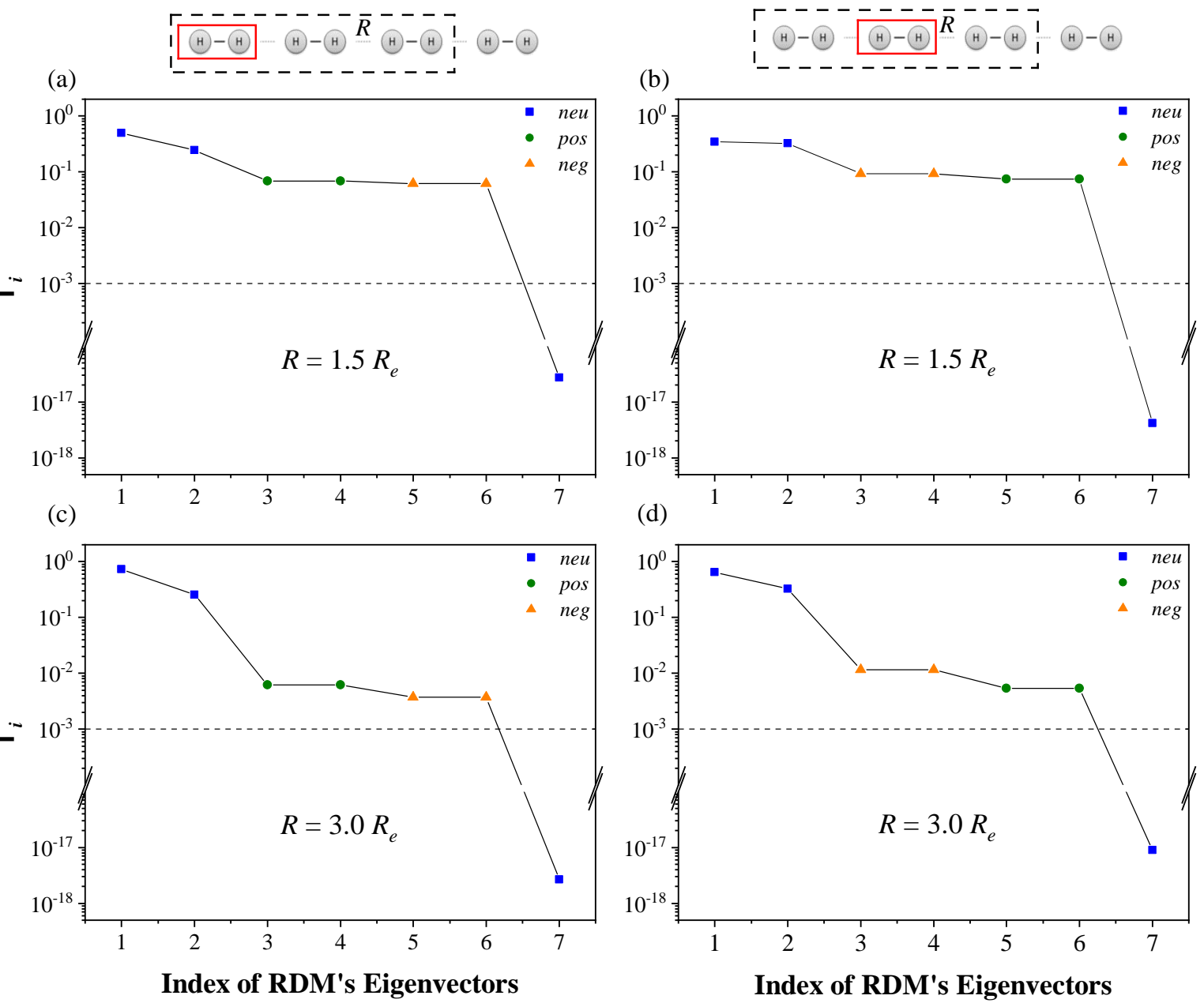

Figure 2: Distribution of RDM's eigenvalues for hydrogen molecules located on edge (a,c) and middle $(\mathrm{b}, \mathrm{d})$ of the tetramer with $R=1.5 / 3.0 R_{e}$ in calculating $\mathrm{T}_{1}$ state under BIPS framework.

Figure 2 shows the distribution of RDM's eigenvalues $\lambda_{i}$ for each block (hydrogen molecule), by taking the hydrogen molecule tetramer as the example (owing to its symmetry only two blocks are plotted). Six RDM eigenvectors including two neu, two pos and two neg are re- 
served with $t_{c}=0.001$ for each block. Their eigenvalues vary with intermolecular distance $R$ changes. $\lambda_{i}$ of ionic eigenvectors (i.e., the pos and neg) in the case of setting $R$ to 1.5 times of the equilibrium bond length of hydrogen molecule $\left(R_{e}, 0.7414 \AA\right)$ are about one order of magnitude larger than the case of $R=3.5 R e$, implying that charge transfer states are non-negligible in short separation distance. It can be noticed that the eigenvalue distribution behaves differently with distinct locations of the monomer in the aggregates. This is mainly attributed to the different chemical environment chosen in calculating the RDM.

Table 1: Calculated $\left(\mathrm{H}_{2}\right)_{n}$ deviations with the intermolecular distance $R=3.0 R_{e}$ by BIPS(2) and BIPS(3) at different tolerance values $t_{c}$ and BIPSs' number $N^{\mathrm{BIPS}}$. Calculations are compared with $\Delta E^{\mathrm{CIS}}\left(\Delta E_{\mathrm{T}_{1}}(4)=15.6352 \mathrm{eV}, \Delta E_{\mathrm{T}_{1}}(8)=15.5969 \mathrm{eV}, \Delta E_{\mathrm{S}_{1}}(4)=23.7140 \mathrm{eV}\right.$ , $\left.\Delta E_{\mathrm{S}_{1}}(8)=23.3740 \mathrm{eV}\right)$.

\begin{tabular}{|c|c|c|c|c|c|}
\hline & \multirow[b]{2}{*}{$t_{c}$} & \multicolumn{2}{|c|}{$\operatorname{BIPS}(2)$} & \multicolumn{2}{|c|}{$\operatorname{BIPS}(3)$} \\
\hline & & $\overline{N^{\mathrm{BIPS}}}$ & $\overline{\delta(2) / \mathrm{meV}}$ & $N^{\mathrm{BIPS}}$ & $\overline{\delta(3) / \mathrm{meV}}$ \\
\hline \multicolumn{6}{|l|}{$\overline{S_{0}-T_{1}}$} \\
\hline \multirow{3}{*}{$\left(\mathrm{H}_{2}\right)_{4}$} & 0.010 & 4 & 413.7 & 4 & 413.7 \\
\hline & 0.005 & 12 & 55.0 & 16 & 51.4 \\
\hline & 0.001 & 16 & 5.6 & 24 & 0.1 \\
\hline \multirow{3}{*}{$\left(\mathrm{H}_{2}\right)_{8}$} & 0.010 & 8 & 451.9 & 8 & 451.9 \\
\hline & 0.005 & 16 & 328.7 & 24 & 326.3 \\
\hline & 0.001 & 36 & 9.4 & 60 & 0.2 \\
\hline \multicolumn{6}{|l|}{$\overline{S_{0}-S_{1}}$} \\
\hline \multirow{3}{*}{$\left(\mathrm{H}_{2}\right)_{4}$} & 0.100 & 4 & 1480.0 & 4 & 1416.1 \\
\hline & 0.030 & 12 & 319.2 & 16 & 191.0 \\
\hline & 0.001 & 16 & 175.7 & 24 & 9.0 \\
\hline \multirow{3}{*}{$\left(\mathrm{H}_{2}\right)_{8}$} & 0.100 & 8 & 1718.9 & 8 & 1611.5 \\
\hline & 0.030 & 32 & 328.3 & 52 & 72.1 \\
\hline & 0.001 & 36 & 308.5 & 60 & 45.9 \\
\hline
\end{tabular}

The automatic identification of important monomer configurations provides an efficient and "block-box" way to produce the localized basis for ab initio exciton models. As an illustration, a series of BIPS(2) calculations are performed by setting different $t_{c}$ values on the hydrogen molecule tetramer and octamer with $R=3.0 R_{e}$. As shown in Table 1 , a steady and monotonic improvement is observed for both excited states $\left(\mathrm{T}_{1}\right.$ and $\left.\mathrm{S}_{1}\right)$ when $t_{c}$ 
value decreases, as well as the growth of $N^{\text {BIPS }}$. An alternative way to improve accuracy is to take into account 3-body interactions. Thence, BIPS(3) calculations are performed for current systems by considering a larger BIPSs' space. Results indicate a vast improvement compared with $\operatorname{BIPS}(2)$, for this reason calculated deviations $\delta(3)$ become smaller than chemical accuracy (i.e., about $43 \mathrm{meV}$ ). In order to further test BIPS performance, given system is extended by setting a series of $R$, to which three low-lying states are calculated using BIPS(2) and $\operatorname{BIPS}(3)\left(t_{c}=0.001\right)$. As can be seen in Figure 3a, BIPS(3) gives proper results in wide range of varying $R$, whereas $\operatorname{BIPS}(2)$ only provides reasonable descriptions in relative long distance. In order to test BIPS ability to describe the aggregation effect, $\operatorname{BIPS}(3)$ calculations $\left(t_{c}=0.001\right)$ from the hydrogen molecule tetramer to decamer are performed. From Figure 3b it is evident that the BIPS(3) performs exceptionally well as plot lines almost perfectly align with the reference lines. The maximum deviation occurs in the calculation of $T_{1}$ for decamer and is only $5.6 \mathrm{meV}$, which is relatively small since the excitation energy of $\mathrm{T}_{1}$ shifts about $102.3 \mathrm{meV}$. It should be pointed out that higher accuracy can be achieved, if necessary, by taking higher many-body interactions into consideration under the BIPS framework.
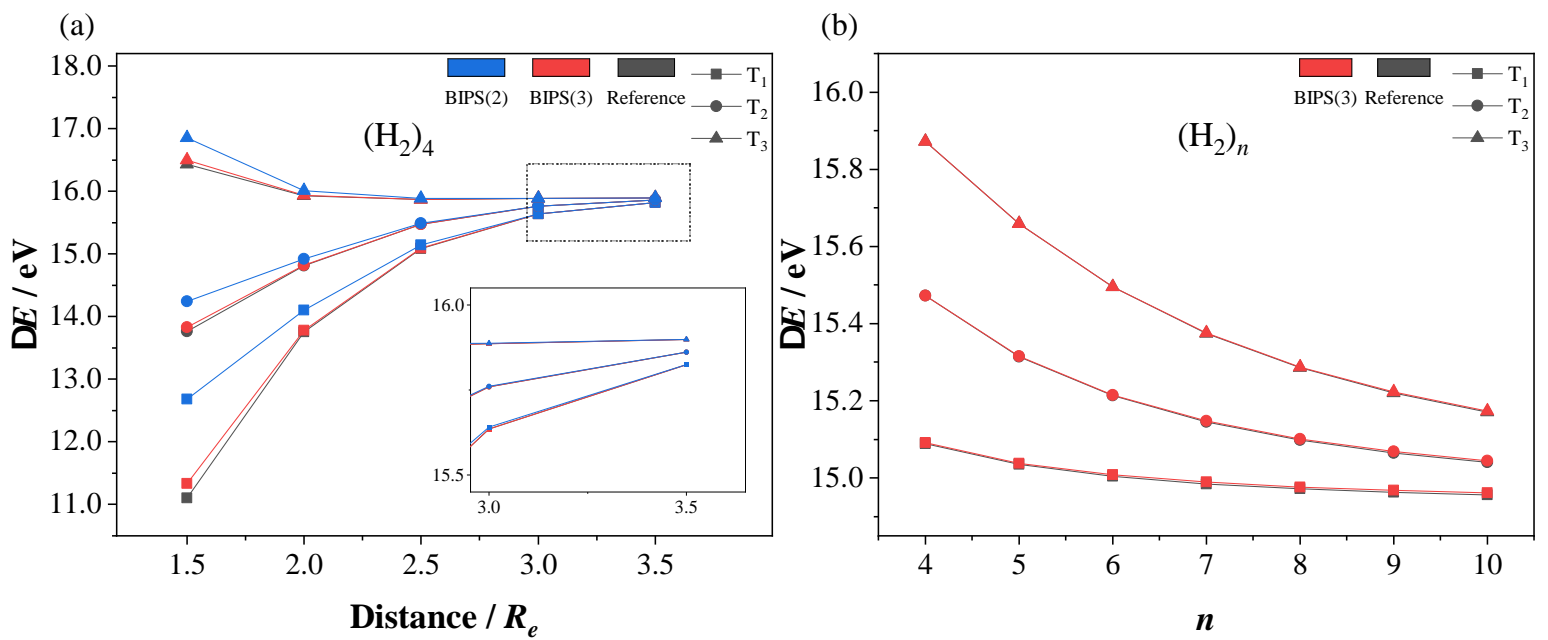

Figure 3: (a): Excitation energies calculated by BIPS(2) and BIPS(3) with hydrogen molecule tetramer with $R$ from $1.5 R_{e}$ to $3.5 R_{e}$, compared with reference. (b): Excitation energies calculated by $\operatorname{BIPS}(3)$ compared with reference from the tetramer to decamer with the fixed $R$ value $2.5 R_{e}$. 
Table 2: Excitation energies deviation for benzene tetramer and octamer calculated by BIPS (counting 2-body effect $\delta(2), 3$-body effect $\delta(3)$ ), compared with the reference. ${ }^{a, b}$

\begin{tabular}{rlccc}
\hline System & & Reference $/ \mathrm{eV}$ & $\delta(2) / \mathrm{meV}$ & $\delta(3) / \mathrm{meV}$ \\
\hline \multirow{3}{*}{$\left(\mathrm{C}_{6} \mathrm{H}_{6}\right)_{4}$} & $\mathrm{~T}_{1}$ & $2.6800(0.5095)$ & 4.6 & 0.3 \\
& $\mathrm{~T}_{2}$ & $2.8672(3.0605)$ & -1.0 & 0.6 \\
& $\mathrm{~T}_{3}$ & $3.0608(2.8668)$ & -2.6 & -2.2 \\
\hline \multirow{3}{*}{$\left(\mathrm{C}_{6} \mathrm{H}_{6}\right)_{8}$} & $\mathrm{~T}_{1}$ & $2.6196(0.5699)$ & 7.0 & -0.3 \\
& $\mathrm{~T}_{2}$ & $2.6749(3.2527)$ & 4.8 & -1.0 \\
& $\mathrm{~T}_{3}$ & $2.7573(3.1704)$ & 1.9 & -2.1 \\
\hline
\end{tabular}

${ }^{a} N^{\mathrm{BIPS}}(4)=52, N^{\mathrm{BIPS}}(8)=120$.

${ }^{b}$ Values in parentheses are the excitation energies' shift compared with $\mathrm{C}_{6} \mathrm{H}_{6}$ monomer (calculated at CIS level).

BIPS performance is further assessed with larger systems - a series of aggregates consisting of different amounts of benzene molecules stacked face-to-face - to verify its computational efficiency. The separation distance between the benzene molecules is fixed at $3.0 \AA$ and detailed geometry structures are given in Table S1 and Table S2. Table 2 contains the results of three low-lying exited states for benzene tetramer and octamer from BIPS(2) and BIPS(3) calculations. As can be seen, both $\operatorname{BIPS}(2)$ and $\operatorname{BIPS}(3)$ provide accurate results, in which deviations are less than $1 \mathrm{kcal} \mathrm{mol}^{-1}$ (about $43 \mathrm{meV}$ ) by an order of magnitude. Consequently, a series of BIPS(2) calculations are performed in benzene aggregates from the tetramer to decamer, results are plotted in Figure 4. For the smallest system, tetramer, the computational time is mainly spent in the construction of BIPSs and the Hamiltonian. With the increasing of system's size, BIPS(2) exhibits almost linear growth in time consumption and almost three times faster than standard CIS calculation in decamer system (shown in Figure 4b). Moreover, calculation accuracy still remains within the maximum deviation of $7 \mathrm{meV}$.

Well-behaved wavefunctions of the excited states are also expected in the calculation under the BIPS framework. Therefore, aggregates consisting of 6 different molecular monomers (i.e., water, ammonia, methanol, methanal, pyridine and ethylene) are studied. Each one of the selected molecules exhibits different type of intermolecular interactions, such as elec- 
(a)

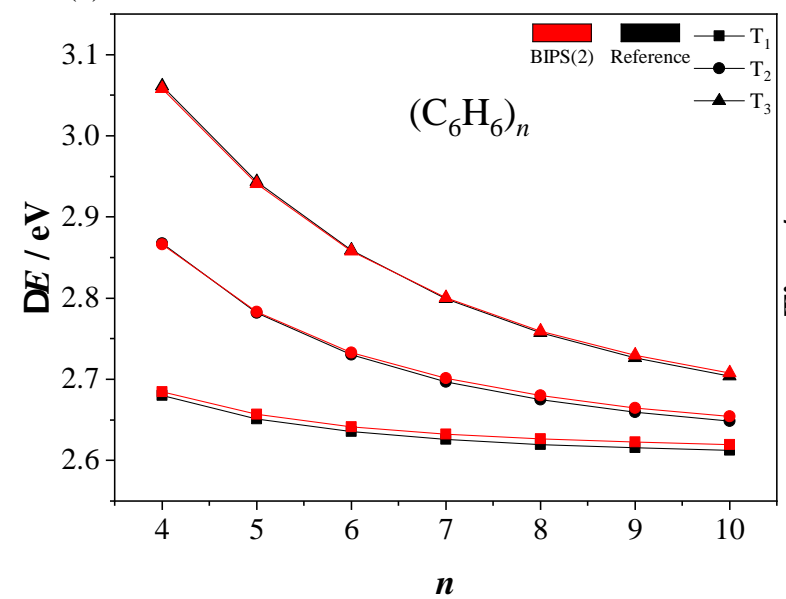

(b)

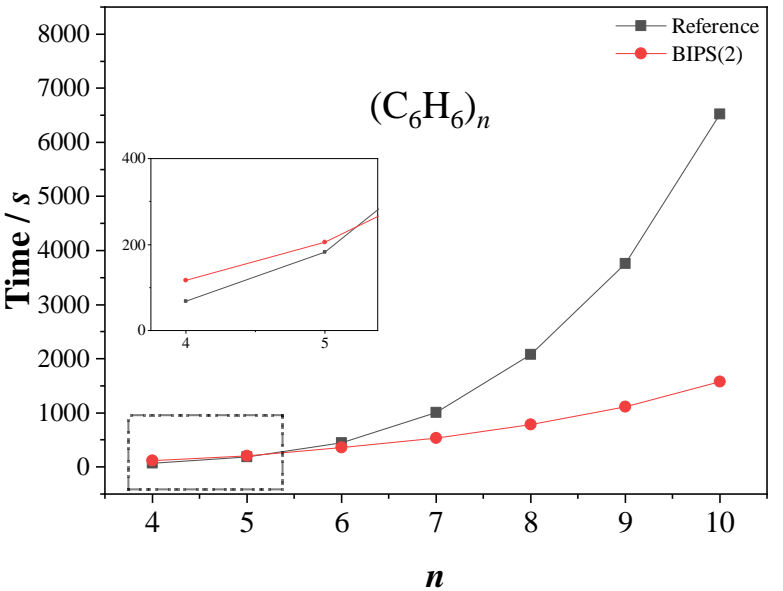

Figure 4: Excited energy (a) and computational costs (b) curves calculated by BIPS(2) as the growth of participating monomer in benzene aggregates with the intermolecular distance $R=3.0 \AA$.

$\mathrm{H}_{2} \mathrm{O}(d=1.85 \AA)$

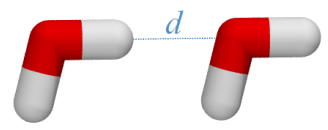

$\mathrm{CH}_{3} \mathrm{OH}(d=2.80 \AA)$

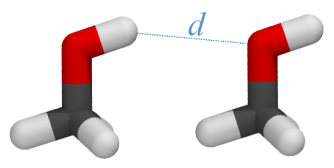

$\mathrm{C}_{5} \mathrm{H}_{5} \mathrm{~N}(d=2.00 \AA)$

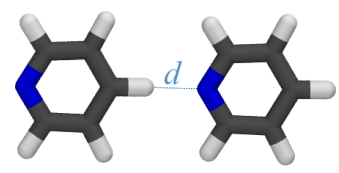

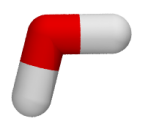
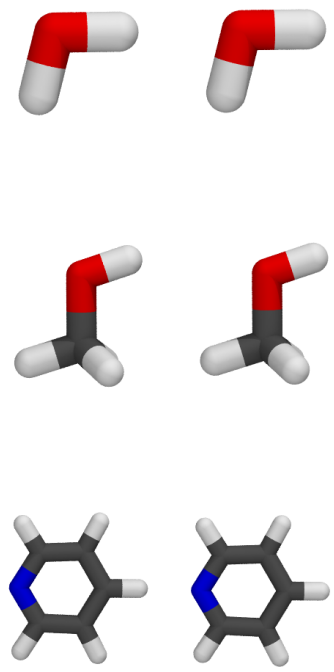

$\mathrm{NH}_{3}(d=2.36 \AA)$
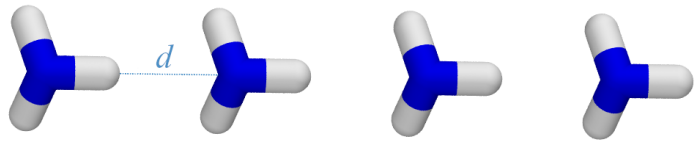

$\mathrm{CH}_{2} \mathrm{O}(d=2.00 \AA)$
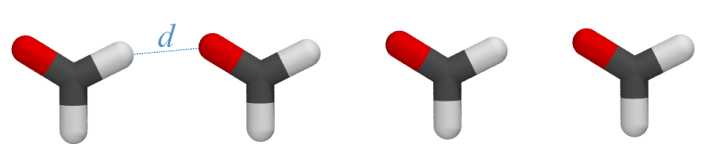

$\mathrm{C}_{2} \mathrm{H}_{4}(d=2.00 \AA)$
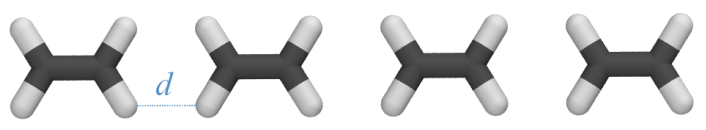

Figure 5: Geometry structures of several molecular aggregates used in BIPS test. 
trostatic (hydrogen bonding) and dispersion (van der Waals). Geometry structures for the selected systems under study are given in Figure 5. Dipole moment $\mu$ of $\mathrm{S}_{1}$, transition dipole moment $\mu^{\mathrm{T}}\left(\mathrm{S}_{0}-\mathrm{S}_{1}\right)$ and excitation energies are calculated by BIPS(2). Computed properties are listed in the Table 3. Results indicate that BIPS(2) gives a relative accurate description of $\mu$ and $\mu^{T}$ with the excitation energies' deviations less than chemical accuracy. Supplementary Table S3 and Table S4 also suggest that the direction of these two properties also fit well with the standard CIS results for most aggregates.

Table 3: Excitation energies, dipole moments $\mu$ of $S_{1}$ and transition dipole moments $\mu^{\mathrm{T}}$ for $\mathrm{S}_{0}-\mathrm{S}_{1}$ calculated by BIPS(2) for a series of aggregates (compared with reference).

\begin{tabular}{|c|c|c|c|c|c|c|}
\hline & \multicolumn{2}{|c|}{$\Delta E / \mathrm{eV}$} & \multicolumn{2}{|c|}{$\mu /$ a.u. } & \multicolumn{2}{|c|}{$\mu^{\mathrm{T}} /$ a.u. } \\
\hline & Reference & $\operatorname{BIPS}(2)$ & Reference & $\operatorname{BIPS}(2)$ & Reference & $\operatorname{BIPS}(2)$ \\
\hline$\left(\mathrm{H}_{2} \mathrm{O}\right)_{4}$ & 12.8927 & 12.9038 & 2.281 & 2.281 & 0.093 & 0.099 \\
\hline$\left(\mathrm{NH}_{3}\right)_{4}$ & 14.9824 & 14.9801 & 1.930 & 1.930 & 0.055 & 0.049 \\
\hline$\left(\mathrm{CH}_{3} \mathrm{OH}\right)_{4}$ & 12.5495 & 12.5349 & 1.674 & 1.667 & 0.141 & 0.130 \\
\hline$\left(\mathrm{CH}_{2} \mathrm{O}\right)_{4}$ & 4.2671 & 4.2749 & 2.242 & 2.233 & 0.010 & 0.009 \\
\hline$\left(\mathrm{C}_{5} \mathrm{H}_{5} \mathrm{~N}\right)_{4}$ & 6.2767 & 6.2681 & 3.281 & 3.282 & 0.266 & 0.271 \\
\hline$\left(\mathrm{C}_{2} \mathrm{H}_{4}\right)_{4}$ & 10.7242 & 10.7430 & 0.000 & 0.000 & 3.426 & 3.336 \\
\hline
\end{tabular}

In summary, we propose a new robust type of bases, block interaction product state (BIPS), to describe the energy and wavefunction of low-lying excited states. BIPS assessment calculations demonstrated that this method is capable of providing accurate descriptions of not only excitation energies, but also first-order properties related to the wavefunctions of excited states. Moreover, BIPS-based method also exhibited low-scaling computational cost. Most compelling evidence has proved its stability for different type of intermolecular interactions. These advantages make BIPS promising for the study of excited states in large practical system, which is part of ongoing research work. BIPS capacity will be further extended by introducing more conventional quantum chemical methods (e.g., CASSCF and TDDFT) and exploring gradient and response properties. 


\section{Acknowledgement}

This work was supported by the National Natural Science Foundation of China (grant number 22073045) and the Fundamental Research Funds for the Central Universities. The authors thank Drs. Luis Vasquez, Zhen Luo and Mr. Yunhao Liu for helpful discussions.

\section{Supporting Information Available}

The following files are available free of charge.

- Implementation of Hamiltonian construction algorithm within the framework of BIPS; supplementary data including atomic coordinates of benzene tetramer and decamer calculated, the components of dipole moment and transition dipole moment for the tetramer of water, ammonia, methanol, methanal, pyridine and ethylene.

\section{References}

(1) Curutchet, C.; Mennucci, B. Quantum Chemical Studies of Light Harvesting. Chem. Rev. 2017, 117, 294-343.

(2) Segatta, F.; Cupellini, L.; Garavelli, M.; Mennucci, B. Quantum Chemical Modeling of the Photoinduced Activity of Multichromophoric Biosystems. Chem. Rev. 2019, 119, 9361-9380.

(3) Mai, S.; González, L. Molecular Photochemistry: Recent Developments in Theory. Angew. Chem. Int. Ed. 2020, 59, 16832-16846.

(4) González, L.; Escudero, D.; Serrano-Andrés, L. Progress and Challenges in the Calculation of Electronic Excited States. ChemPhysChem 2011, 13, 28-51. 
(5) Mester, D.; Kállay, M. Reduced-Scaling Approach for Configuration Interaction Singles and Time-Dependent Density Functional Theory Calculations Using Hybrid Functionals. J. Chem. Theory Comput. 2019, 15, 1690-1704.

(6) He, X.; Zhu, T.; Wang, X.; Liu, J.; Zhang, J. Z. H. Fragment Quantum Mechanical Calculation of Proteins and Its Applications. Acc. Chem. Res. 2014, 47, 2748-2757.

(7) Collins, M. A.; Bettens, R. P. A. Energy-Based Molecular Fragmentation Methods. Chem. Rev. 2015, 115, 5607-5642.

(8) Raghavachari, K.; Saha, A. Accurate Composite and Fragment-Based Quantum Chemical Models for Large Molecules. Chem. Rev. 2015, 115, 5643-5677.

(9) Herbert, J. M. Fantasy versus reality in fragment-based quantum chemistry. J. Chem. Phys. 2019, 151, 170901.

(10) Li, W.; Dong, H.; Ma, J.; Li, S. Structures and Spectroscopic Properties of Large Molecules and Condensed-Phase Systems Predicted by Generalized Energy-Based Fragmentation Approach. Acc. Chem. Res. 2021, 54, 169-181.

(11) Fedorov, D. G.; Kitaura, K. Multiconfiguration self-consistent-field theory based upon the fragment molecular orbital method. J. Chem. Phys. 2005, 122, 054108.

(12) Kaliakin, D. S.; Nakata, H.; Kim, Y.; Chen, Q.; Fedorov, D. G.; Slipchenko, L. V. FMOxFMO: Elucidating Excitonic Interactions in the Fenna-Matthews-Olson Complex with the Fragment Molecular Orbital Method. J. Chem. Theory Comput. 2020, $16,1175-1187$.

(13) Li, W.; Li, Y.; Lin, R.; Li, S. Generalized Energy-Based Fragmentation Approach for Localized Excited States of Large Systems. J. Phys. Chem. A 2016, 120, 9667-9677.

(14) Jin, X.; Glover, W. J.; He, X. Fragment Quantum Mechanical Method for Excited 
States of Proteins: Development and Application to the Green Fluorescent Protein. J. Chem. Theory Comput. 2020, 16, 5174-5188.

(15) Chen, W.-K.; Fang, W.-H.; Cui, G. Integrating Machine Learning with the Multilayer Energy-Based Fragment Method for Excited States of Large Systems. J. Phys. Chem. Lett. 2019, 10, 7836-7841.

(16) Macetti, G.; Genoni, A. Quantum Mechanics/Extremely Localized Molecular Orbital Embedding Strategy for Excited States: Coupling to Time-Dependent Density Functional Theory and Equation-of-Motion Coupled Cluster. J. Chem. Theory Comput. 2020, 16, 7490-7506.

(17) Zhang, W.; Liu, J.; Jin, X.; Gu, X.; Zeng, X. C.; He, X.; Li, H. Quantitative Prediction of Aggregation-Induced Emission: A Full Quantum Mechanical Approach to the Optical Spectra. Angew. Chem. Int. Ed. 2020, 59, 11550-11555.

(18) Wu, F.; Liu, W.; Zhang, Y.; Li, Z. Linear-Scaling Time-Dependent Density Functional Theory Based on the Idea of From Fragments to Molecule. J. Chem. Theory Comput. 2011, 7, 3643-3660.

(19) Yam, C.; Yokojima, S.; Chen, G. Linear-scaling time-dependent density-functional theory. Phys. Rev. B 2003, 68, 153105.

(20) Cui, G.; Fang, W.; Yang, W. Reformulating time-dependent density functional theory with non-orthogonal localized molecular orbitals. Phys. Chem. Chem. Phys. 2010, 12, $416-421$.

(21) Liu, J.; Herbert, J. M. An efficient and accurate approximation to time-dependent density functional theory for systems of weakly coupled monomers. J. Chem. Phys. 2015, 143, 034106. 
(22) Macetti, G.; Genoni, A. Quantum Mechanics/Extremely Localized Molecular Orbital Embedding Strategy for Excited States: Coupling to Time-Dependent Density Functional Theory and Equation-of-Motion Coupled Cluster. J. Chem. Theory Comput. 2020, 16, 7490-7506.

(23) Baseggio, O.; Fronzoni, G.; Stener, M. A new time dependent density functional algorithm for large systems and plasmons in metal clusters. J. Chem. Phys. 2015, 143, 024106 .

(24) Liu, J.; Hu, W.; Yang, J. Two-level iterative solver for linear response time-dependent density functional theory with plane wave basis set. J. Chem. Phys. 2021, 154, 064101.

(25) Nakatsuji, H.; Miyahara, T.; Fukuda, R. Symmetry-adapted-cluster/symmetryadapted-cluster configuration interaction methodology extended to giant molecular systems: Ring molecular crystals. J. Chem. Phys. 2007, 126, 084104.

(26) Chwee, T. S.; Carter, E. A. Valence Excited States in Large Molecules via Local Multireference Singles and Doubles Configuration Interaction. J. Chem. Theory Comput. 2011, 7, 103-111.

(27) Crawford, T. D.; King, R. A. Locally correlated equation-of-motion coupled cluster theory for the excited states of large molecules. Chem. Phys. Lett. 2002, 366, 611-622.

(28) Kats, D.; Korona, T.; Schütz, M. Local CC2 electronic excitation energies for large molecules with density fitting. J. Chem. Phys. 2006, 125, 104106.

(29) Krisiloff, D. B.; Carter, E. A. Approximately size extensive local multireference singles and doubles configuration interaction. Phys. Chem. Chem. Phys. 2012, 14, 7710.

(30) Das, A.; Müller, T.; Plasser, F.; Krisiloff, D. B.; Carter, E. A.; Lischka, H. Local Electron Correlation Treatment in Extended Multireference Calculations: Effect of 
Acceptor-Donor Substituents on the Biradical Character of the Polycyclic Aromatic Hydrocarbon Heptazethrene. J. Chem. Theory Comput. 2017, 13, 2612-2622.

(31) Ye, H.-Z.; Tran, H. K.; Voorhis, T. V. Accurate Electronic Excitation Energies in FullValence Active Space via Bootstrap Embedding. J. Chem. Theory Comput. 2021, 17, $3335-3347$.

(32) Baudin, P.; Bykov, D.; Liakh, D.; Ettenhuber, P.; Kristensen, K. A local framework for calculating coupled cluster singles and doubles excitation energies (LoFEx-CCSD). Mol. Phys. 2017, 115, 2135-2144.

(33) Frank, M. S.; Hättig, C. A pair natural orbital based implementation of CCSD excitation energies within the framework of linear response theory. J. Chem. Phys. 2018, 148,134102 .

(34) Frank, M. S.; Schmitz, G.; Hättig, C. Implementation of the iterative triples model CC3 for excitation energies using pair natural orbitals and Laplace transformation techniques. J. Chem. Phys. 2020, 153, 034109.

(35) Dutta, A. K.; Saitow, M.; Demoulin, B.; Neese, F.; Izsák, R. A domain-based local pair natural orbital implementation of the equation of motion coupled cluster method for electron attached states. J. Chem. Phys. 2019, 150, 164123.

(36) Baseggio, O.; Vetta, M. D.; Fronzoni, G.; Stener, M.; Sementa, L.; Fortunelli, A.; Calzolari, A. Photoabsorption of Icosahedral Noble Metal Clusters: An Efficient TDDFT Approach to Large-Scale Systems. J. Phys. Chem. C 2016, 120, 12773-12782.

(37) Yoshikawa, T.; Komoto, N.; Nishimura, Y.; Nakai, H. GPU-Accelerated Large-Scale Excited-State Simulation Based on Divide-and-Conquer Time-Dependent DensityFunctional Tight-Binding. J. Comput. Chem. 2019, 40, 2778-2786. 
(38) Yoshikawa, T.; Yoshihara, J.; Nakai, H. Large-scale excited-state calculation using dynamical polarizability evaluated by divide-and-conquer based coupled cluster linear response method. J. Chem. Phys. 2020, 152, 024102.

(39) Nakai, H.; Yoshikawa, T. Development of an excited-state calculation method for large systems using dynamical polarizability: A divide-and-conquer approach at the timedependent density functional level. J. Chem. Phys. 2017, 146, 124123.

(40) Lim, S.-H.; Bjorklund, T. G.; Spano, F. C.; Bardeen, C. J. Exciton Delocalization and Superradiance in Tetracene Thin Films and Nanoaggregates. Phys. Rev. Lett. 2004, 92, 107402.

(41) Hestand, N. J.; Kazantsev, R. V.; Weingarten, A. S.; Palmer, L. C.; Stupp, S. I.; Spano, F. C. Extended-Charge-Transfer Excitons in Crystalline Supramolecular Photocatalytic Scaffolds. J. Am. Chem. Soc. 2016, 138, 11762-11774.

(42) Zhao, H.; Zhao, Y.; Song, Y.; Zhou, M.; Lv, W.; Tao, L.; Feng, Y.; Song, B.; Ma, Y.; Zhang, J. et al. Strong optical response and light emission from a monolayer molecular crystal. Nat. Commun. 2019, 10, 5589.

(43) Green, J. A.; Asha, H.; Santoro, F.; Improta, R. Excitonic Model for Strongly Coupled Multichromophoric Systems: The Electronic Circular Dichroism Spectra of Guanine Quadruplexes as Test Cases. J. Chem. Theory Comput. 2021, 17, 405-415.

(44) Morrison, A. F.; Herbert, J. M. Low-Scaling Quantum Chemistry Approach to ExcitedState Properties via an ab Initio Exciton Model: Application to Excitation Energy Transfer in a Self-Assembled Nanotube. J. Phys. Chem. Lett. 2015, 6, 4390-4396.

(45) Herbert, J. M.; Zhang, X.; Morrison, A. F.; Liu, J. Beyond Time-Dependent Density Functional Theory Using Only Single Excitations: Methods for Computational Studies of Excited States in Complex Systems. Acc. Chem. Res. 2016, 49, 931-941. 
(46) Hsu, C.-P. The Electronic Couplings in Electron Transfer and Excitation Energy Transfer. Acc. Chem. Res. 2009, 42, 509-518.

(47) Subotnik, J. E.; Alguire, E. C.; Ou, Q.; Landry, B. R.; Fatehi, S. The Requisite Electronic Structure Theory To Describe Photoexcited Nonadiabatic Dynamics: Nonadiabatic Derivative Couplings and Diabatic Electronic Couplings. Acc. Chem. Res. 2015, 48, 1340-1350.

(48) Zang, H.; Zhao, Y.; Liang, W. Quantum Interference in Singlet Fission: J- and HAggregate Behavior. J. Phys. Chem. Lett. 2017, 8, 5105-5112.

(49) Wang, Y.-C.; Feng, S.; Liang, W.; Zhao, Y. Electronic Couplings for Photoinduced Charge Transfer and Excitation Energy Transfer Based on Fragment Particle-Hole Densities. J. Phys. Chem. Lett. 2021, 12, 1032-1039.

(50) Wen, J.; Ma, H. A fragmentation-based approach for evaluating the intra-chain excitonic couplings in conjugated polymers. Chem. Phys. Lett. 2017, 679, 152-157.

(51) Li, X.; Parrish, R. M.; Liu, F.; Schumacher, S. I. L. K.; Martínez, T. J. An Ab Initio Exciton Model Including Charge-Transfer Excited States. J. Chem. Theory Comput. 2017, 13, 3493-3504.

(52) Canola, S.; Bagnara, G.; Dai, Y.; Ricci, G.; Calzolari, A.; Negri, F. Addressing the Frenkel and charge transfer character of exciton states with a model Hamiltonian based on dimer calculations: Application to large aggregates of perylene bisimide. J. Chem. Phys. 2021, 154, 124101.

(53) Wilson, K. G. Renormalization Group and Critical Phenomena. I. Renormalization Group and the Kadanoff Scaling Picture. Phys. Rev. B 1971, 4, 3174-3183.

(54) Morningstar, C. J.; Weinstein, M. Contractor Renormalization Group Method: A New Computational Technique for Lattice Systems. Phys. Rev. Lett. 1994, 73, 1873-1877. 
(55) Parker, S. M.; Seideman, T.; Ratner, M. A.; Shiozaki, T. Communication: Active-space decomposition for molecular dimers. J. Chem. Phys. 2013, 139, 021108.

(56) Parker, S. M.; Shiozaki, T. Communication: Active space decomposition with multiple sites: Density matrix renormalization group algorithm. J. Chem. Phys. 2014, 141, 211102.

(57) Hajj, M. A.; Malrieu, J.-P.; Guihéry, N. Renormalized excitonic method in terms of block excitations: Application to spin lattices. Phys. Rev. B 2005, 72, 224412.

(58) Zhang, H.; Malrieu, J.-P.; Ma, H.; Ma, J. Implementation of renormalized excitonic method at ab initio level. J. Comput. Chem. 2011, 33, 34-43.

(59) Ma, Y.; Liu, Y.; Ma, H. A new fragment-based approach for calculating electronic excitation energies of large systems. J. Chem. Phys. 2012, 136, 024113.

(60) Ma, Y.; Ma, H. Calculating Excited States of Molecular Aggregates by the Renormalized Excitonic Method. J. Phys. Chem. A 2013, 117, 3655-3665.

(61) Liu, Y.; Wang, K.; Ma, H. Evaluating First-Order Molecular Properties of Delocalized Ionic or Excited States in Molecular Aggregates by Renormalized Excitonic Method. Chinese J. Chem. Phys. 2021, In Press.

(62) Nishio, S.; Kurashige, Y. Rank-one basis made from matrix-product states for a lowrank approximation of molecular aggregates. J. Chem. Phys. 2019, 151, 084110.

(63) Schollwöck, U. The density-matrix renormalization group. Rev. Mod. Phys. 2005, 77, 259-315.

(64) White, S. R. Density matrix formulation for quantum renormalization groups. Phys. Rev. Lett. 1992, 69, 2863-2866.

(65) Zhao, Y.; Truhlar, D. G. The M06 suite of density functionals for main group thermochemistry, thermochemical kinetics, noncovalent interactions, excited states, and 
transition elements: two new functionals and systematic testing of four M06-class functionals and 12 other functionals. Theor. Chem. Acc. 2008, 120, 215-241.

(66) Frisch, M. J.; Trucks, G. W.; Schlegel, H. B.; Scuseria, G. E.; Robb, M. A.; Cheeseman, J. R.; Scalmani, G.; Barone, V.; Petersson, G. A.; Nakatsuji, H. et al. Gaussian16 Revision C.01. 2016; Gaussian Inc. Wallingford CT.

(67) Galván, I. F.; Vacher, M.; Alavi, A.; Angeli, C.; Aquilante, F.; Autschbach, J.; Bao, J. J.; Bokarev, S. I.; Bogdanov, N. A.; Carlson, R. K. et al. OpenMolcas: From Source Code to Insight. J. Chem. Theory Comput. 2019, 15, 5925-5964.

(68) Aquilante, F.; Autschbach, J.; Baiardi, A.; Battaglia, S.; Borin, V. A.; Chibotaru, L. F.; Conti, I.; Vico, L. D.; Delcey, M.; Galván, I. F. et al. Modern quantum chemistry with [Open]Molcas. J. Chem. Phys. 2020, 152, 214117. 


\section{TOC Graphic}

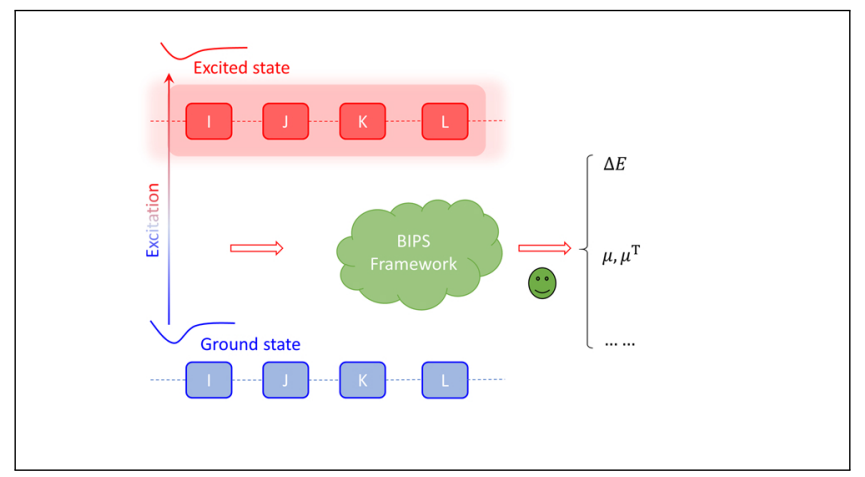

